\title{
Giant Coronary Artery Aneurysm due to Polyarteritis Nodosa in an Eight-Year-Old Child
}

\section{Olga Cecilia Maza, MD1, Carlos Alberto Gallon, MD², Rafael Vicente Echeverria, MD², Cristina Isabel Morales, MD ${ }^{4}$ and John Jairo Araujo, MBBS, MASt, MD, MSIAC ${ }^{5^{*}}$ (D)}

\author{
${ }^{1}$ Pediatric Cardiologist, Department of Pediatric Cardiology, Northern General Clinical Organization, \\ Barranquilla, Colombia \\ ${ }^{2}$ Pediatric Coordinator and Intensive Care Pediatrician, Department of Northern General Clinical Organization, \\ Barranquilla, Colombia \\ ${ }^{3}$ Pediatric Hemodynamicist, Department of Pediatric Cardiology, Northern General Clinical Organization, \\ Barranquilla, Colombia
}

${ }^{4}$ Pediatrician, Department of Pediatrics, Renacer Clinic, Barranquilla, Colombia

${ }^{5}$ Cardiologist Echocardilogist in Pediatric and Adult Congenital Heart Disease, Department of Clinic and Research in Cardiology, SOMA Clinic, Medellin, Colombia

*Corresponding author: John Jairo Araujo, MBBS, MASt, MD, MSIAC, Cardiologist Echocardilogist in Pediatric and Adult Congenital Heart Disease, Department of Clinic and Research in Cardiology, SOMA Clinic, Medellin, 050015, Colombia, Tel: +575768651

\begin{abstract}
Systemic polyarteritis nodosa is a vasculitis characterized by inflammation and fibrinoid necrosis of medium and small arteries, with a global incidence of $0.7 / 100,000$ and a prevalence of $6.3 / 100,000$. Its cause is as yet unknown, and it affects both children and adults. The clinical presentation tends to be insidious and vague in its initial stages. Cardiac involvement is one of the signs of severity, with coronary artery impairment and aneurysmal lesions of varying degrees. The consequent heart failure and acute myocardial infarction are complications which increase mortality. Without treatment, five-year survival is $13 \%$. Medical treatment is aimed at halting the inflammatory process and treating the consequences of the dysfunction of various organs and systems. We present a case of an eight-year-old boy with polyarteritis nodosa who developed giant aneurysms of the coronary arteries with severe cardiac dysfunction and multisystem impairment. The proper diagnosis and prompt treatment achieved clinical recovery and early survival.
\end{abstract}

\section{Keywords}

Polyarteritis nodosa, Coronary artery aneurysms, Multisystemic vasculitis, Necrotizing vasculitis

\section{Introduction}

Polyarteritis nodosa (PAN) was first described in 1852 by the German pathologist from Vienna, Karl von Rokitansky, in a 23-year-old man with a five-day history of fever and diarrhea [1]. It is a systemic necrotizing vasculitis characterized by inflammation and fibrinoid necrosis of medium and small arteries [2]. It has a global incidence of $0.7 / 100,000$ and a prevalence of $6.3 / 100,000[3,4]$. Its etiology is unknown, but it has been related to various infectious processes. Between $10-50 \%$ of adult cases are related to the hepatitis B virus, and some are related to the hepatitis $C$ virus. Case reports in children are scarce [5]. In 2004, Ozen, et al. published a multicenter review with 110 pediatric patients, finding a relationship with hepatitis $B$ and $C$ in only $4.6 \%$ of cases [6]. Typically, it presents as a systemic process, characterized by fever and general malaise, asthenia, anorexia, fatigue, and weight loss. Various organs and systems (cardiovascular, renal, gastrointestinal, musculoskeletal, and nervous system, among others) are affected. Local and systemic manifestations

Citation: Maza OC, Gallon CA, Echeverria RV, Morales Cl, Araujo JJ (2021) Giant Coronary Artery Aneurysm due to Polyarteritis Nodosa in an Eight-Year-Old Child. Int J Clin Cardiol 8:221. doi. org/10.23937/2378-2951/1410221

Accepted: March 18, 2021: Published: March 20, 2021

Copyright: (c) 2021 Maza OC, et al. This is an open-access article distributed under the terms of the Creative Commons Attribution License, which permits unrestricted use, distribution, and reproduction in any medium, provided the original author and source are credited. 


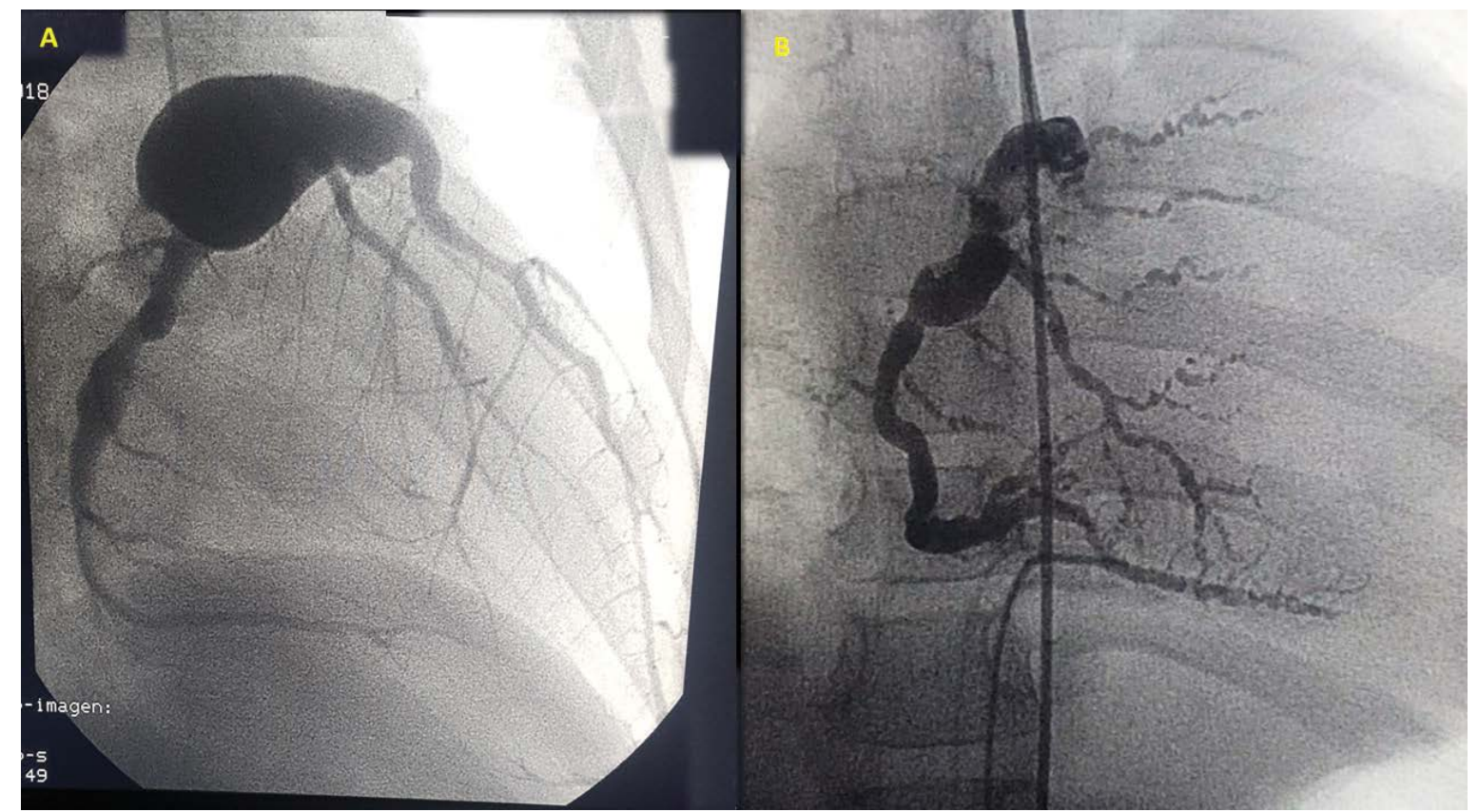

Figure 1: $(A)$ Coronary catheterization showing a giant aneurysm affecting the left main coronary artery; (B) Right coronary artery, with multiple aneurysmal dilations (the aneurysmal rosary sign).

depend on the disease severity and organ involvement, and include maculopapular purpuric lesions which may be complicated by necrotic lesions and peripheral skin gangrene, as well as proteinuria and glomerulonephritis which may progress to kidney failure [7]. From a cardiovascular standpoint, PAN may affect the coronary arteries (CAs), with the development of aneurysmal lesions of varying sizes which progress to heart failure (HF), acute myocardial infarction, myocarditis, and conduction disorders, among others [8-10].

\section{Case Report}

This was an eight-year-old male patient who had been hospitalized nine months before due to prolonged fever, maculopapular skin lesions on his upper limbs, myalgia, arthralgias and peripheral edema. He had leukocytosis and elevated C-reactive protein (CRP). His echocardiogram was normal. Kawasaki disease (KD) was suspected, and he received intravenous immunoglobulin in addition to intravenous penicillin for suspected sepsis. His symptoms improved and he was discharged after 30 days. He did not report any monitoring or follow up. He was referred to our cardiovascular center with a two-month history of deteriorating functional class (FC), weight loss, intermittent fever $\left(39^{\circ} \mathrm{C}\right)$, and progressive dyspnea. On physical exam, his heart rate was $138 / \mathrm{min}$, respiratory rate $32 / \mathrm{min}$, blood pressure $100 / 60 \mathrm{mmHg}$, temperature $37{ }^{\circ} \mathrm{C}$, weight $26 \mathrm{~kg}$, and height $160 \mathrm{~cm}$, with enlarged neck and inguinal lymph nodes. His heart had a regular rhythm, with low-pitched first and second sounds and no murmurs. A chest X-ray showed cardiomegaly. An electrocardiogram showed sinus tachycardia with no signs of ischemia. An echocardiogram showed a large pericardial effusion, left ventricular (LV) dilation, and severe systolic dysfunction with an ejection fraction
(EF) of 33\%; the CAs could not be evaluated. A pericardial window was done, extracting $350 \mathrm{~mL}$ of clear liquid (transudate) whose culture and immunological studies were negative. The clinical deterioration persisted (hepatomegaly, edema of the lower limbs and systemic arterial hypertension [SAH]), with ongoing leukocytosis and neutrophilia, elevated erythrocyte sedimentation rate and CRP, as well as proteinuria and ascites, and no improvement with medical treatment for $\mathrm{HF}$.

A heart catheterization revealed a giant aneurysm of the left main coronary artery (LCA) and right coronary artery (RCA), with multiple aneurysmal dilations (the aneurysmal rosary sign) (Figure 1 and Video 1). In addition, there were diffuse small vessel lesions on the distal pulmonary artery, and mesenteric and renal arteries (Figure 2 and Video 1). Thus, systemic PAN was diagnosed. Intravenous corticosteroids, cyclophosphamide and platelet antiaggregation (acetylsalicylic acid + clopidogrel) were administered. He was also treated for HF with carvedilol, enalapril, furosemide and spironolactone. Follow up one year later showed a normal-sized LV, $60 \% \mathrm{EF}$, and persistent lesions in the CAs. The proximal third of the LCA was $19.2 \mathrm{~mm} \times 18.1 \mathrm{~mm}$, and the RCA was $13.9 \mathrm{~mm} \times 14.2 \mathrm{~mm}$. Angiography of the renal and pulmonary vessels showed that the lesions had resolved (Figure 3). He was in $\mathrm{FCl}$, with no acute ischemic events.

\section{Discussion}

As a multisystemic vasculitis, the clinical presentation of systemic PAN may range from a self-limited disease with no sequelae to fulminant multisystem failure. Most cases are of the serious type [11]. The classic presentation in childhood is insidious, with symptoms which include SAH in up to $80 \%$ of cases, fever, general 


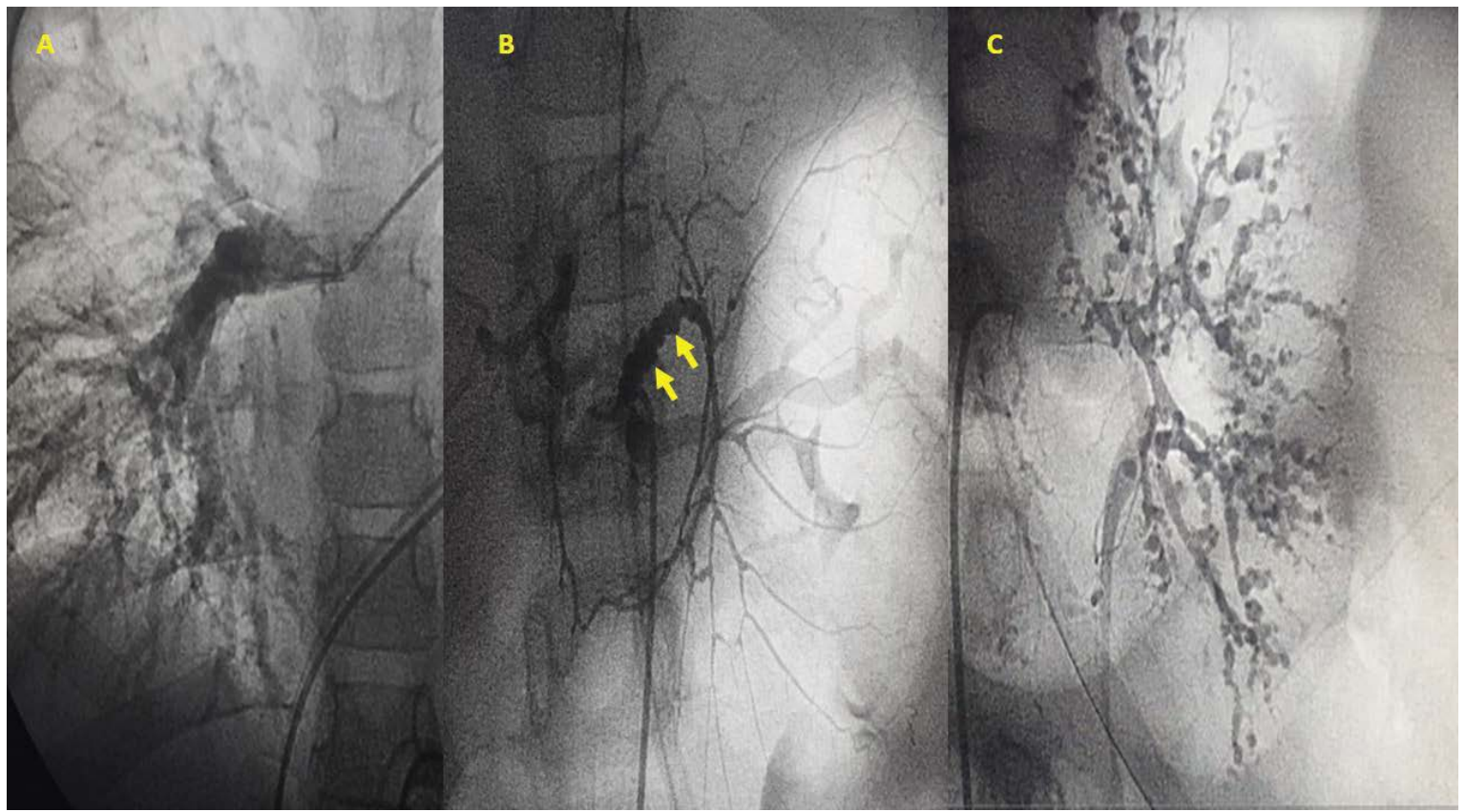

Figure 2: (A) Pulmonary artery angiography showing diffuse small vessel lesions on the distal right pulmonary artery; (B) Catheterization of the abdominal aorta; mesenteric angiography shows multiple saccular vascular lesions (yellow arrows); (C) Left renal catheterization; angiography shows diffuse small vessel lesions.

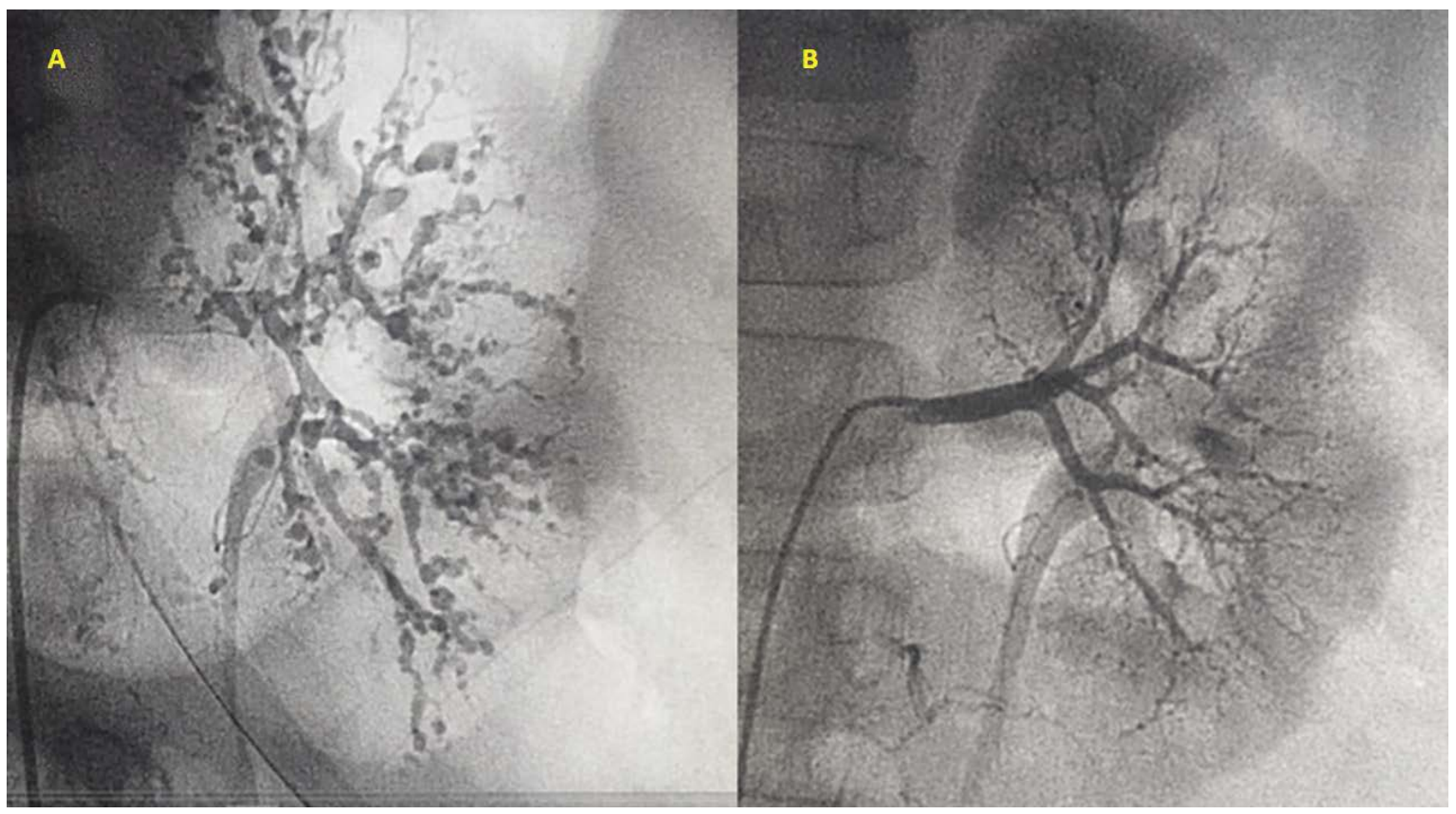

Figure 3: Comparative angiography of the left renal arteries: (A) Initial lesions and (B) After successful treatment and one year of follow up.

malaise, fatigue, anorexia, weight loss, myalgia, muscular sensitivity, eye symptoms and testicular pain $[12,13]$. Medium-vessel vasculitides include PAN, cutaneous polyarteritis and KD. These vasculitides have common symptoms such as persistent fever and constitutional symptoms like weight loss, myalgia and arthralgias. These symptoms are accompanied by purpuric skin lesions and altered lab tests indicative of inflammation such as leukocytosis, eosinophilia, increased erythrocyte sedimentation rate, and elevated CRP, among others. Systemic signs of dysfunction will depend on the organs and systems (cardiovascular, renal, digestive, musculoskeletal) involved. Untreated KD, specifically, may affect the CAs in $15-25 \%$ of cases, with the development of aneurysms which may lead to myocardial infarction, sudden death or ischemic heart disease [14]. The patient's first assessment was performed outside of our institution. We postulate that the physicians thought of a possible KD due to the previously described symptoms, and decided to administer intravenous immunoglobulin treatment. This may have caused transient symptom resolution and delayed the PAN diagnosis. However, in 
our institutional assessment, we no found past history about bilateral conjunctivitis, inflammation of mucous membranes of the mouth and throat (dry, red, cracked lips and a strawberry-red tongue) swelling of lymph nodes in the neck (cervical lymphadenopathy); redness and swelling of the hands and feet, peeling skin on the hands and feet. We propose the hypothesis that, given the findings at our cardiovascular center, PAN may have begun from the onset of symptoms. Although KD has some symptoms in common with PAN, it is clear that its occurrence is uncommon in children over the age of five [15].

Heart involvement in systemic PAN is unusual, and occurs in 5 to $20 \%$ of cases, generally in the context of other organ and system involvement. Coronary artery aneurysms occur most often in the RCA, followed by the left anterior descending artery and, finally, the circumflex; they rarely involve both main arteries and their divisions $[16,17]$. In the presented case, we found a giant LCA aneurysm on the main trunk, associated with an RC aneurysm. This explains the HF which developed prior to being admitted to our institution. Despite the giant size of the LC aneurysm, the CAs could not be assessed in the first echocardiographic study due to the large pericardial effusion and persistent tachycardia which made a detailed evaluation difficult. However, when HF is present, various congenital abnormalities of the coronary circulation must be ruled out (anomalous left coronary artery from the pulmonary artery [ALCAPA]). However, there were no signs of ischemia on the electrocardiogram, which made the diagnosis more difficult. Systemic arterial hypertension is a very common finding, but in our case it was not found initially, due to great hemodynamic instability and signs of imminent cardiac tamponade secondary to the large pericardial effusion (as evidenced by tachycardia and normal arterial pressure). Once the pericardial effusion resolved, SAH was revealed, providing another diagnostic criterion for systemic PAN. In light of the unexplained cause of HF, coronary angiography was key for diagnosing the CA lesions. The characteristic sign of an "aneurysmal rosary" is described in the context of multisystem involvement, and is often seen in the mesenteric arteries in systemic PAN; this was found in the RC (Figure 2). Kawasaki disease, Behçet's disease, Takayasu's arteritis, giant cell arteritis or syphilitic arteritis, and ALCAPA should be ruled out in the differential diagnosis.

Systemic PAN is diagnosed according to the criteria proposed by the European League Against Rheumatism/ Pediatric Rheumatology International Trials Organisation/Pediatric Rheumatology European Society (EULAR/ PRINTO/PRES), respectively, which identify necrotizing vasculitis when at least one minor criterion is present along with an angiographic anomaly such as an aneurysm, stenosis or occlusion of a medium or small artery The diagnosis of systemic PAN has a sensitivity and spe- cificity of $89.6 \%$ and $99.6 \%$, respectively [18]. The case presented met the classification criteria for a diagnosis of systemic PAN (SAH, non-nephrotic proteinuria and myalgias), together with multiple disorders of the coronary and renal vessels.

Currently, the recommended treatment of choice for systemic PAN aims to decrease systemic vascular inflammation. The administration of steroids combined with cyclophosphamide or azathioprine has a greater than $80 \%$ effectiveness [19]. Suggested alternatives include methotrexate, mycophenolate mofetil, immunoglobulin, infliximab or rituximab. However, these results are based on studies in adults. In our case, there was a good response to corticosteroids and cyclophosphamide, associated with good HF control. However, after successful treatment, the disease recurs in $10-20 \%$ of patients [20]. Likewise, when CA aneurysms are present, platelet antiaggregation is mandatory [21].

Without treatment, the five-year survival is $13 \%$. With optimal treatment, five-year survival increases approximately $80 \%$, with a mortality of approximately $1-4 \%[22]$.

\section{Conclusions}

Systemic PAN is a rare, progressive disease with an insidious presentation. The prognosis is determined by the involvement of multiple organs and systems. This case highlights a significant involvement of the CAs, developing giant aneurysms and significantly affecting cardiac function. Due to the insidious presentation, the diagnosis tends to be unclear and may be delayed, as in this case. It is important to differentiate PAN from other types of vasculitis, including KD. The diagnostic guidelines proposed by EULAR/PRINTO/PRES must be followed to avoid delayed diagnosis and treatment. This caseteaches us that, in the presence of $\mathrm{HF}$ or fever of unknown origin, and once the more common diseases are ruled out, systemic PAN must be suspected. Angiographic diagnosis is definitive for detecting CA lesions, and treatment aimed at stopping the inflammation and avoiding greater consequences should be promptly instated. Between $10-20 \%$ of systemic PAN patients relapse; thus, specialized follow up is necessary, including complementary CA imaging, as well as antiplatelet therapy.

\section{References}

1. Tesar V, Kazderová M, Hlavácková L (2004) Rokitansky and his first description of polyarteritis nodosa. J Nephrol 17: $172-174$

2. Kawakami T (2012) A review of pediatric vasculitis with a focus on juvenile polyarteritis nodosa. Am J Clin Dermatol 13: $389-398$

3. Mossberg M, Segelmark M, Kahn R, Englund M, Mohammad AJ (2018) Epidemiology of primary systemic vasculitis in children: A population-based study from southern Sweden. Scand J Rheumatol 47: 295-302. 
4. Barut K, Sahin S, Kasapcopur O (2016) Pediatric vasculitis. Curr Opin Rheumatol 28: 29-38.

5. Eleftheriou D, Brogan PA (2018) Vasculitis in children. Paediatr Child Health 28: 57-63.

6. Ozen S, Anton J, Arisoy N, Bakkaloglu A, Besbas N, et al. (2004) Juvenile polyarteritis: Results of a multicenter survery of 110 children. J Pediatr 145: 517-522.

7. Eleftheriou D, Dillon MJ, Tullus K, Marks SD, Pilkington CA, et al. (2013) Systemic polyarteritis nodosa in the young: A single-center experience over thirty-two years. Arthritis Rheum 65: 2476-2485.

8. Holsinger DR, Osmundson PJ, Edwards JE (1962) The heart in periarteritis nodosa. Circulation 25: 610-618.

9. Peters B, von Spiczak J, Ruschitzka F, Distler O, Manka R, et al. (2018) Cardiac manifestation of polyarteritis nodosa. Eur Heart J 39: 2603.

10. Wi J, Choi HH, Lee CJ, Kim T, Shin S, et al. (2010) Acute myocardial infarction due to polyarteritis nodosa in a young female patient. Korean Circ J 40: 197-200.

11. Schrader ML, Hochman JS, Bulkley BH (1985) The heart in polyarteritis nodosa: A clinicopathologic study. Am Heart $\mathrm{J}$ 109: 1353-1359.

12. Mogale KD, Shrivastava A (2006) Childhood polyarteritis nodosa: A clinical diagnosis. Indian Pediatr 43: 911-913.

13. Khanna G, Sargar K, Baszis KW (2015) Pediatric vasculitis: Recognizing multisystemic manifestations at body imaging. Radiographics 35: 849-865.

14. Galeotti C, Bayry J, Kone-Paut I, Kaveri SV (2010) Kawasaki disease: Aetiopathogenesis and therapeutic utility of intravenous immunoglobulin. Autoimmun Rev 9: 441-448.
15. Moreno E, Garcia SD, Bainto E, Salgado AP, Parish A, et al. (2020) Presentation and outcomes of kawasaki disease in Latin American infants younger than 6 months of age: A multinational multicenter study of the REKAMLATINA Network. Front Pediatr 8: 384.

16. Miloslavsky E, Unizony S (2014) The heart in vasculitis. Rheum Dis Clin North Am 40: 11-26.

17. EIGuindy MS, EIGuindy AM (2017) Aneurysmal coronary artery disease: An overview. Glob Cardiol Sci Pract 2017: e201726.

18. Ozen S, Pistorio A, Iusan SM, Bakkaloglu A, Herlin T, et al. (2010) EULAR/PRINTO/PRES criteria for henoch- schönlein purpura, childhood polyarteritis nodosa, childhood wegener granulomatosis and childhood takayasu arteritis: Ankara 2008. Part II: Final classification criteria. Ann Rheum Dis 69: 798-806.

19. Gedalia A, Cuchacovich R (2009) Systemic vasculitis in childhood. Curr Rheumatol Rep 11: 402-409.

20. Glanz S, Bittner SJ, Berman MA, Dolan Jr TF, Talner NS (1976) Regression of coronary-artery aneurysms in infantile polyarteritis nodosa. N Engl J Med 294: 939-941.

21. Sheikh AS, Hailan A, Kinnaird T, Choudhury A, Smith D (2019) Coronary artery aneurysm: Evaluation, prognosis, and proposed treatment strategies. Heart Views 20: 101108.

22. Gayraud M, Guillevin L, le Toumelin P, Cohen P, Lhote F, et al. (2001) Long-term followup of polyarteritis nodosa, microscopic polyangiitis, and churg-strauss syndrome: Analysis of four prospective trials including 278 patients. Arthritis Rheum 44: 666-675. 